\title{
THE $U V J$ SELECTION OF QUIESCENT AND STAR-FORMING GALAXIES: SEPARATING EARLY- AND LATE-TYPE GALAXIES AND ISOLATING EDGE-ON SPIRALS ${ }^{*, \dagger, \ddagger}$
}

\author{
Shannon G. Patel ${ }^{1}$, Bradford P. Holden ${ }^{2}$, Daniel D. Kelson ${ }^{3}$, Marijn Franx ${ }^{1}$, \\ ArJen VAN DER WEL ${ }^{4}$, AND GARTh D. IllingWORTH ${ }^{2}$ \\ ${ }^{1}$ Leiden Observatory, Leiden University, P.O. Box 9513, NL-2300 AA Leiden, The Netherlands; patel@ strw.leidenuniv.nl \\ 2 UCO/Lick Observatory, University of California, Santa Cruz, CA 95064, USA \\ ${ }^{3}$ Observatories of the Carnegie Institution of Washington, Pasadena, CA 91101, USA \\ ${ }^{4}$ Max-Planck-Institut für Astronomie, Königstuhl 17, D-69117 Heidelberg, Germany \\ Received 2011 July 14; accepted 2012 February 28; published 2012 March 15
}

\begin{abstract}
We utilize for the first time Hubble Space Telescope Advanced Camera for Surveys imaging to examine the structural properties of galaxies in the rest-frame $U-V$ versus $V-J$ diagram (i.e., the $U V J$ diagram) using a sample at $0.6<z<0.9$ that reaches a low stellar mass limit $\left(\log M / M_{\odot}>10.25\right)$. The use of the $U V J$ diagram as a tool to distinguish quiescent galaxies from star-forming galaxies (SFGs) is becoming more common due to its ability to separate red quiescent galaxies from reddened SFGs. Quiescent galaxies occupy a small and distinct region of $U V J$ color space and we find most of them to have concentrated profiles with high Sérsic indices $(n>2.5)$ and smooth structure characteristic of early-type systems. SFGs populate a broad but well-defined sequence of $U V J$ colors and are comprised of objects with a mix of Sérsic indices. Interestingly, most $U V J$-selected SFGs with high Sérsic indices also display structure due to dust and star formation typical of the $n<2.5$ SFGs and late-type systems. Finally, we find that the position of an SFG on the sequence of $U V J$ colors is determined to a large degree by the mass of the galaxy and its inclination. Systems that are closer to edge-on generally display redder colors and lower [O II] $] 3727$ luminosity per unit mass as a consequence of the reddening due to dust within the disks. We conclude that the two main features seen in $U V J$ color space correspond closely to the traditional morphological classes of early- and late-type galaxies.
\end{abstract}

Key words: galaxies: evolution - galaxies: formation - galaxies: structure

Online-only material: color figures

\section{INTRODUCTION}

In the absence of morphological information to distinguish early- and late-type galaxies, the use of UV-optical colors has emerged as a commonly employed proxy in studies of galaxy evolution. This practice is bolstered by the color bimodality (e.g., Strateva et al. 2001; Baldry et al. 2004). For example, galaxies are often divided in a color-magnitude or color-mass diagram into red and blue, and the evolution in the luminosity or mass function of each color type tracked over cosmic time (e.g., Bell et al. 2004; Faber et al. 2007; Brown et al. 2007, and several others). The underlying assumption in the method above is that the red galaxies represent quiescent systems with little or no ongoing star formation (SF). However, it is well known that a reddened star-forming galaxy (SFG) can display colors coincident with those on the red sequence (e.g., Maller et al. 2009). Selecting red galaxies based on a single rest-frame color, in combination with a magnitude or stellar mass, therefore results in a selection of both quiescent galaxies and reddened SFGs. This presents a predicament for studies that use such a selection in order to quantify the evolution in the number of passive systems.

\footnotetext{
* This Letter includes data gathered with the $6.5 \mathrm{~m}$ Magellan Telescopes located at Las Campanas Observatory, Chile.

$\dagger$ Based in part on data collected at Subaru Telescope and obtained from the SMOKA, which is operated by the Astronomy Data Center, National Astronomical Observatory of Japan.

$\ddagger$ Based on observations made with the NASA/ESA Hubble Space Telescope, obtained at the Space Telescope Science Institute. STScI is operated by the Association of Universities for Research in Astronomy, Inc. under NASA contract NAS 5-26555.
}

In this Letter, we highlight the utility of a color-color diagram, specifically rest-frame $U-V$ versus $V-J$ (hereafter referred to as the $U V J$ diagram), in distinguishing quiescent galaxies from SFGs, including those SFGs that are heavily reddened. The $U V J$ diagram is gaining increasing visibility in studies of galaxy evolution (e.g., Labbé et al. 2005; Wuyts et al. 2007; Williams et al. 2009, 2010; Brammer et al. 2009, 2011; Whitaker et al. 2010; Patel et al. 2011; Quadri et al. 2012). In Patel et al. (2011) we hinted at the possibility of the $U V J$ selection of quiescent and SFGs being used to also distinguish morphologically earlyand late-type systems. Here we introduce for the first time an analysis based on Hubble Space Telescope (HST) Advanced Camera for Surveys (ACS) imaging and examine the structural properties of galaxies in $U V J$ color space in order to address the prospects of using two rest-frame colors in classifying both the recent star formation histories (SFHs) and morphologies of galaxies.

We use a stellar mass-limited sample of galaxies at $0.6<$ $z<0.9$ to carry out our analysis. As is well known, many galaxy properties correlate with stellar mass and it is therefore crucial that one control for stellar mass in interpreting results. The redshift range examined here provides a suitable case study for a sample of galaxies in the distant universe. The rest-frame $J$-band magnitudes at these redshifts represent observed $K_{s}$, which is typically the reddest bandpass accessible from the ground for deep wide-field galaxy surveys.

We assume a cosmology with $H_{0}=70 \mathrm{~km} \mathrm{~s}^{-1} \mathrm{Mpc}^{-1}$, $\Omega_{M}=0.30$, and $\Omega_{\Lambda}=0.70$. Stellar masses are based on a Chabrier initial mass function (Chabrier 2003). All magnitudes are reported in the $\mathrm{AB}$ system. 


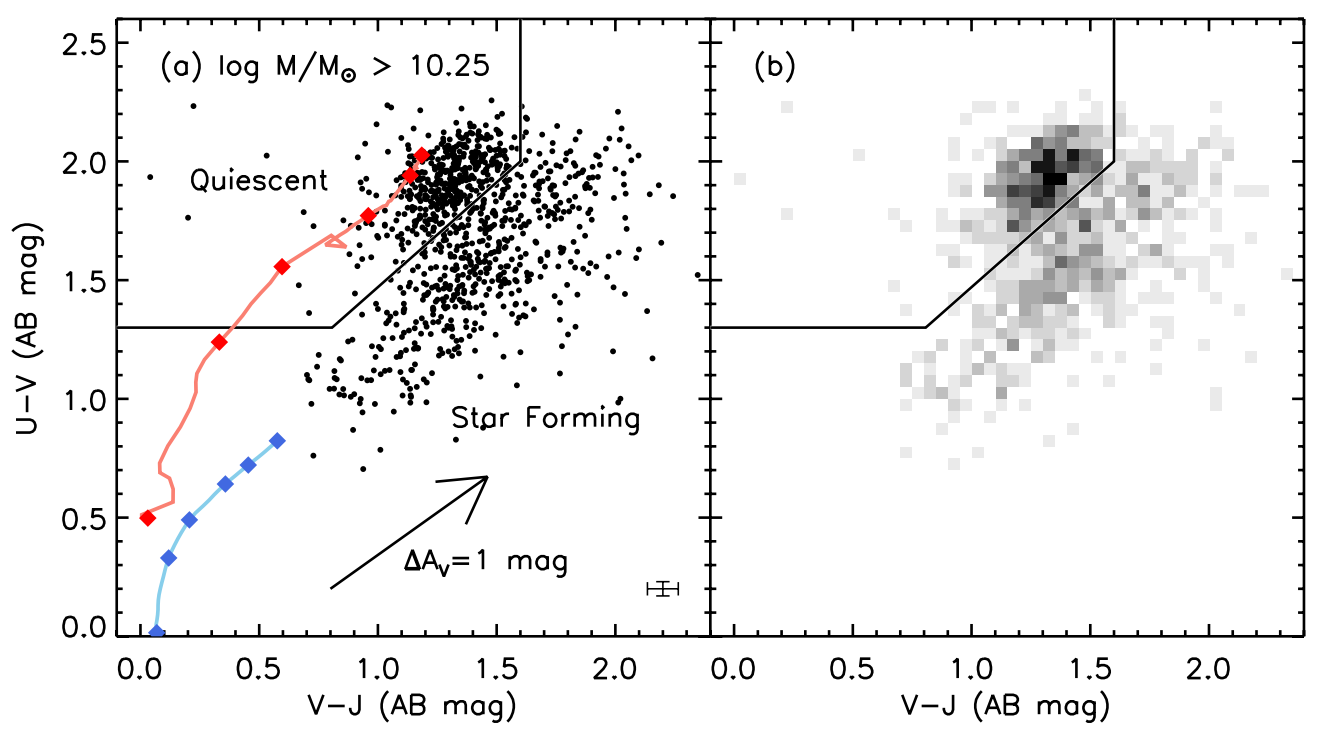

Figure 1. (a) Rest-frame $U-V$ vs. $V-J$ colors for 977 galaxies with mass $\log M / M_{\odot}>10.25$ at $0.6<z<0.9$. The typical $\pm 1 \sigma$ statistical uncertainty in the rest-frame colors is indicated in the bottom right. The Williams et al. (2009) boundary (black wedge) separates quiescent galaxies (top left) from SFGs (bottom right). The red line indicates the evolution of a BC03 solar metallicity single burst, while the blue line represents a constant SFR model (CSF). The diamonds on each model track represent time steps of $0.1,0.5,1,2,3$, and $5 \mathrm{Gyr}$ (from bottom to top). The arrow indicates the reddening vector. (b) Grayscale representation of the density of points in panel (a). The concentration of quiescent galaxies in the $U V J$ plane is distinct from the broader distribution of SFGs.

(A color version of this figure is available in the online journal.)

\section{DATA AND ANALYSIS}

Data products for our sample are discussed in detail in Patel et al. (2009a, 2009b, 2011) and briefly summarized here. Ground-based imaging consists of Magellan IMACS $B$, Subaru Suprime-Cam $V R i z$, and Du Pont WIRC $K_{s}$. The $z$ band was used for spectroscopic selection $\left(18<z_{\mathrm{AB}}<23.3 \mathrm{mag}\right)$. We obtained spectroscopy with IMACS on Magellan, using a low-dispersion prism (LDP) in place of the grating. Roughly, $\sim 10,000$ redshifts were measured over a $\sim 0.2 \mathrm{deg}^{2}$ field. The overall spectroscopic completeness is $\sim 74 \%$. Our sample in this work is restricted to galaxies in the redshift range $0.6<z<0.9$, with the lower redshift cutoff determined by the bright magnitude limit of our survey and the upper redshift cutoff determined by the desired mass cut and with some consideration for the redshift precision. In this redshift interval, the LDP redshifts have a precision of $\sigma_{z} /(1+z) \approx 0.012$ when compared to redshifts obtained from higher resolution spectroscopy. We find 3326 galaxies in this redshift interval with $z_{\mathrm{AB}}<23.3 \mathrm{mag}$. We fit Bruzual \& Charlot (2003, hereafter referred to as BC03) $\tau$-models to spectral energy distributions (SEDs) comprised of both the broadband photometry and LDP spectra in order to extract relevant rest-frame quantities such as stellar masses. The limiting stellar mass for constructing representative samples of both star-forming and passive galaxies is determined by the latter and is $M>1.8 \times 10^{10} M_{\odot}\left(\log M / M_{\odot}>10.25\right)$ at $z=0.9$. The SED fitting procedure also provides measurements of [O II] 3727 luminosities. Rest-frame magnitudes and colors were computed with the ground-based imaging and using the $K$-correction technique described in Rudnick et al. (2003).

We use HST/ACS imaging (Ford et al. 2003) to measure the structural properties of galaxies. About $\sim 50 \%$ of our masslimited sample with $K_{s}$ imaging has ACS coverage in either the F775W or F814W filter. Although the ACS imaging targeted galaxies in slightly higher density environments, we do not expect this to significantly impact our key conclusions. The color postage stamps shown in the next section are for the subset of our sample with ACS coverage in two filters (i.e., either F606W and F814W, or F625W and F775W). We used GALFIT (Peng et al. 2002) on the F775W or F814W imaging to measure axis ratios, $b / a$, Sérsic indices, $n$, and effective radii, $R_{e}$. We direct the reader to Holden et al. (2009) for a detailed discussion on the fitting procedure as well as results from simulations pertaining to the reliability of the axis-ratio measurements. We also measured the bumpiness parameter, $B$, which provides an estimate of the higher order structure within galaxies and is defined as the ratio of the rms of the residual from the GALFIT model fit and the model mean (see, e.g., Blakeslee et al. 2006; van der Wel 2008).

Our final mass-limited sample at $0.6<z<0.9$ with $K_{s}$ imaging and measured structural parameters from HST imaging is comprised of 487 galaxies.

\section{THE $U V J$ DIAGRAM}

\subsection{Selecting Quiescent and SFGs}

In Figure 1 we present the rest-frame $U V J$ diagram for a stellar mass-limited sample at $0.6<z<0.9$. The combination of these two colors allows one to break the degeneracy between age and reddening for red galaxies as discussed below. Quiescent galaxies are delineated from SFGs using the boundary computed by Williams et al. (2009). We note that this boundary results in $\sim 31 \%$ of galaxies with $U-V>1.7 \mathrm{AB}$ mag being classified as SFGs. This fraction does not vary significantly for different mass bins above our mass cut. Figure 1(b) shows a grayscale representation of the density of points in the $U V J$ diagram. The concentration of quiescent galaxies is distinct from the broader distribution of $U V J$ classified SFGs.

Model SFHs reinforce the Williams et al. (2009) division between quiescent and SFGs in the UVJ diagram. The color evolution of a BC03 solar metallicity single burst (SSP) and constant star formation (CSF) rate model are shown in Figure 1(a). After $~ 3-5$ Gyr, the SSP lies in the region of the $U V J$ diagram populated by quiescent galaxies. Meanwhile, after $\sim 5 \mathrm{Gyr}$, the CSF lies in the star-forming region of the $U V J$ diagram where no galaxies with mass $M>1.8 \times 10^{10} M_{\odot}$ can 


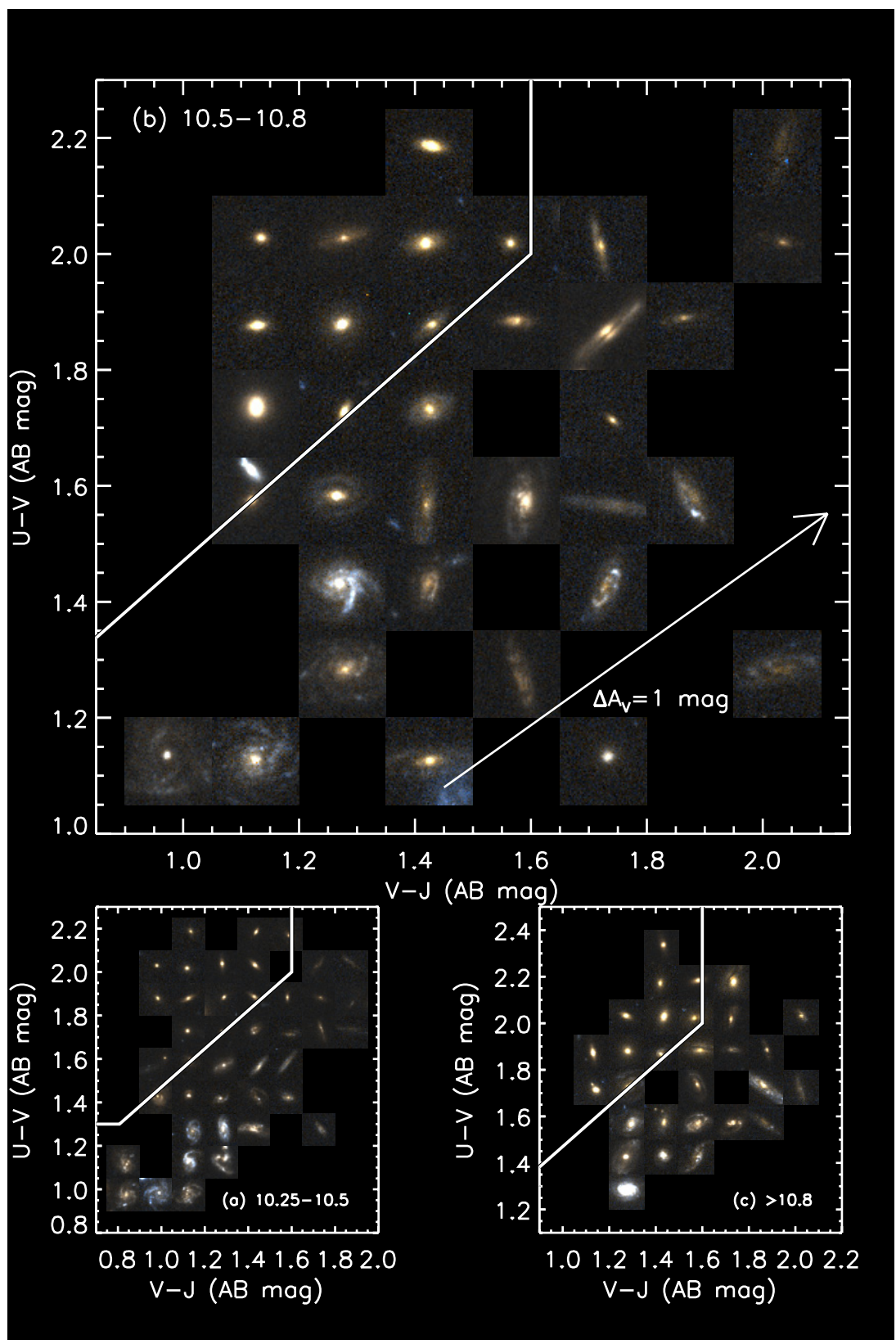

Figure 2. Rest-frame $U V J$ diagram at $0.6<z<0.9$ represented with $H S T / \mathrm{ACS}$ color postage stamps (either F606W and F814W or F625W and F775W, $3^{\prime \prime}$ on a side) for galaxies in three stellar mass bins: (a) $10.25<\log M / M_{\odot}<10.5$, (b) $10.5<\log M / M_{\odot}<10.8$, and (c) $\log M / M_{\odot}>10.8$. In all mass bins, $U V J$-selected quiescent galaxies are mostly early-type systems while SFGs are primarily late-type systems. Bluer SFGs are generally face-on disks, while redder ones tend to be viewed edge-on.

(A color version of this figure is available in the online journal.)

be found. However, the reddening vector, computed assuming a Calzetti et al. (2000) reddening law, suggests that the addition of varying amounts of extinction can explain a large range of colors for SFGs above our mass limit. Patel et al. (2011) show the color evolution of more complex SFHs in the $U V J$ diagram, including bursts and other composite SFHs. We reiterate their finding that the slightest amount of SF on top of a dominant older stellar population can easily move galaxies from the quiescent region to the star-forming region. The SSP and CSF models shown here are intended to provide a simple overview of the kinds of SFHs that can produce colors consistent with $U V J$ classified quiescent and SFGs.

Finally, Patel et al. (2011) showed that MIPS $24 \mu \mathrm{m}$ detections primarily trace the region of the $U V J$ diagram occupied by SFGs including those with the reddest colors $(U-V>$ 1.7 AB mag), therefore providing empirical support for the Williams et al. (2009) boundary.

\subsection{The Morphological Structure of Galaxies in the UVJ Diagram}

With $H S T$ /ACS imaging, we gain a new understanding for the kinds of galaxies that populate various regions of the $U V J$ diagram. These data provide perhaps the most informative explanation as to why the $U V J$ selection works as well as it does. In Figure 2, for three different mass bins, we show the $U V J$ diagram populated with $H S T /$ ACS color postage stamps for the subset of galaxies with ACS coverage. Each color-color 


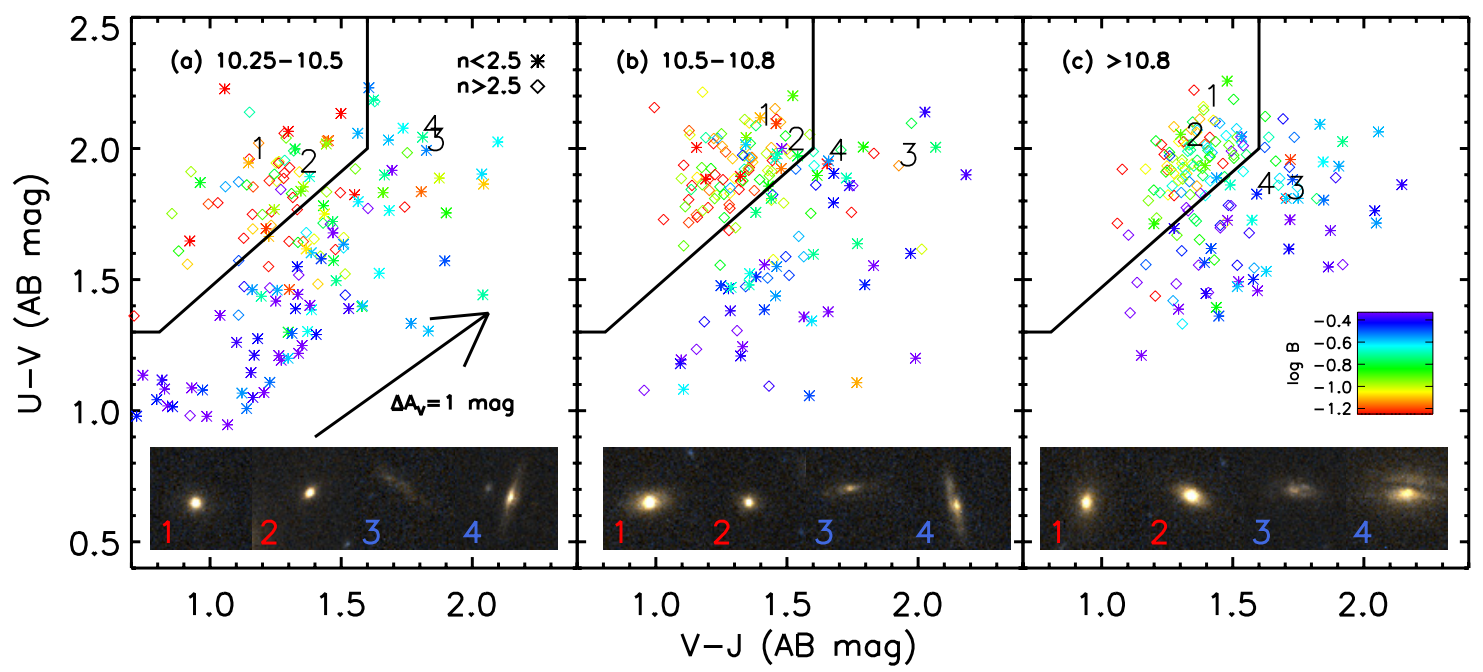

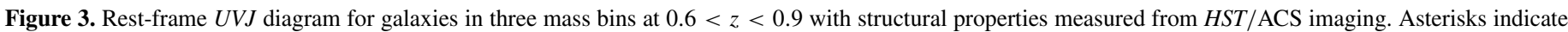

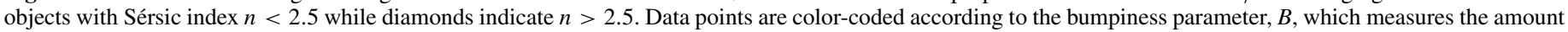

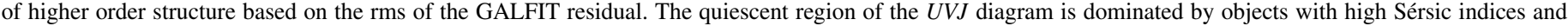

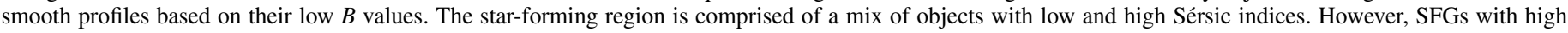

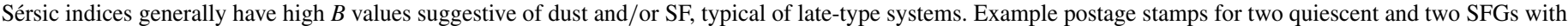

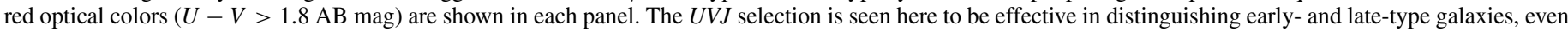
on the red sequence.

(A color version of this figure is available in the online journal.)

bin ( $0.15 \mathrm{mag}$ in size $)$ is represented with a postage stamp of a random galaxy within the bin. These images display rest-frame light at $\sim 3000-5000 \AA$, similar to the wavelength range probed by the $U-V$ color. The contrast in structure between galaxies selected in the $U V J$ diagram to be quiescent versus star forming is striking. The quiescent region is populated primarily by earlytype galaxies. In contrast, the star-forming region is dominated by late-type galaxies.

In Figure 3, we look more quantitatively at the structural properties of galaxies in the $U V J$ diagram for the same three mass bins as Figure 2. The symbols indicate whether galaxies have $n<2.5$ (asterisks) or $n>2.5$ (diamonds) and are color-coded according to the bumpiness value, $B$. Galaxies with higher order structure, suggestive of dust and/or SF in late-type systems, have higher values of $B$.

In the quiescent region of the $U V J$ diagram, the fraction of galaxies with $n>2.5$ for the low-, intermediate-, and highmass bin is $71 \% \pm 6 \%, 85 \% \pm 4 \%$, and $93 \% \pm 3 \%$, respectively. We note that most of the quiescent galaxies with $n<2.5$ generally have low $B$ values, indicating featureless profiles. The somewhat higher fraction at lower masses generally reside in more overdense environments relative to $n<2.5$ SFGs, and could therefore represent quenched satellites. Thus, in all mass bins, the quiescent region is indeed dominated by galaxies with concentrated and/or featureless surface brightness profiles typical of early-type galaxies.

In the star-forming region of the $U V J$ diagram, the proportion of galaxies that have $n<2.5$ for the low-, intermediate-, and high-mass bin is $74 \% \pm 4 \%, 58 \% \pm 6 \%$, and $46 \% \pm 6 \%$, respectively. Thus, a substantial fraction of SFGs are not best fitted with low Sérsic indices indicative of disk-dominated systems, especially at higher masses. However, for those SFGs with $n>2.5$, most also have high values for $B$ indicating the presence of higher order structure common to late-type galaxies. These galaxies generally have strong bulges, likely responsible for driving the Sérsic fit, but also a visually identifiable disk component with strong dust and/or SF, a key difference from the $n>2.5$ quiescent systems. Thus, the quiescent and star- forming regions of the $U V J$ diagram have been shown here to correspond closely to the traditional morphological classes of early- and late-type galaxies.

We note that the models of Tuffs et al. (2004) predict that high-opacity, star-forming disks viewed near edge-on can contaminate the quiescent region of the $U V J$ diagram. While highly inclined $(b / a<0.4)$ disk-dominated galaxies do exist in this region, they generally have higher Sérsic indices and smoother light profiles (i.e., low $B$ values) than similarly inclined galaxies in the star-forming region of the $U V J$ diagram. These differences in structure may be attributed to higher opacity or the inclined galaxies in the quiescent region are indeed lacking SF activity and are typical S0 galaxies (see, e.g., Holden et al. 2011).

\section{INCLINATION: A KEY SOURCE FOR THE SEQUENCE OF UVJ COLORS OF SFGs}

As previously noted, SFGs span a wide range of colors in the $U V J$ diagram. There are two possibilities for the source of this diversity in the $U V J$ colors at a fixed mass: (1) differences in the amount of reddening and/or (2) differences in the SFHs. Metallicity is not expected to be a significant factor, given that at a fixed mass, the variation in metallicity is only $\sim \pm 0.1 \mathrm{dex}$ (Tremonti et al. 2004). For a CSF model, this variation in metallicity would result in a negligible change in the $U-V$ color and a change of only $\sim \pm 0.1 \mathrm{mag}$ in the $V-J$ color, which is not enough to explain the broad range of $U V J$ colors for SFGs. We focus on option (1) above in this Letter and will show that this component can account for a significant portion of the variation in $U V J$ colors for SFGs. In fact, Figure 2 hints at a trend between the viewing inclination of SFGs, which is related to the degree of reddening, and $U V J$ colors. While bluer SFGs appear to be viewed closer to face-on, redder SFGs are more likely to be viewed closer to edge-on. This is apparent for all mass bins.

Figure 4 reinforces the interpretation that the colors of SFGs are driven to a large degree by the viewing angle. Figure 4(a) shows the axis ratio, $b / a$, versus $V-J$, for SFGs with $n<2.5$. 


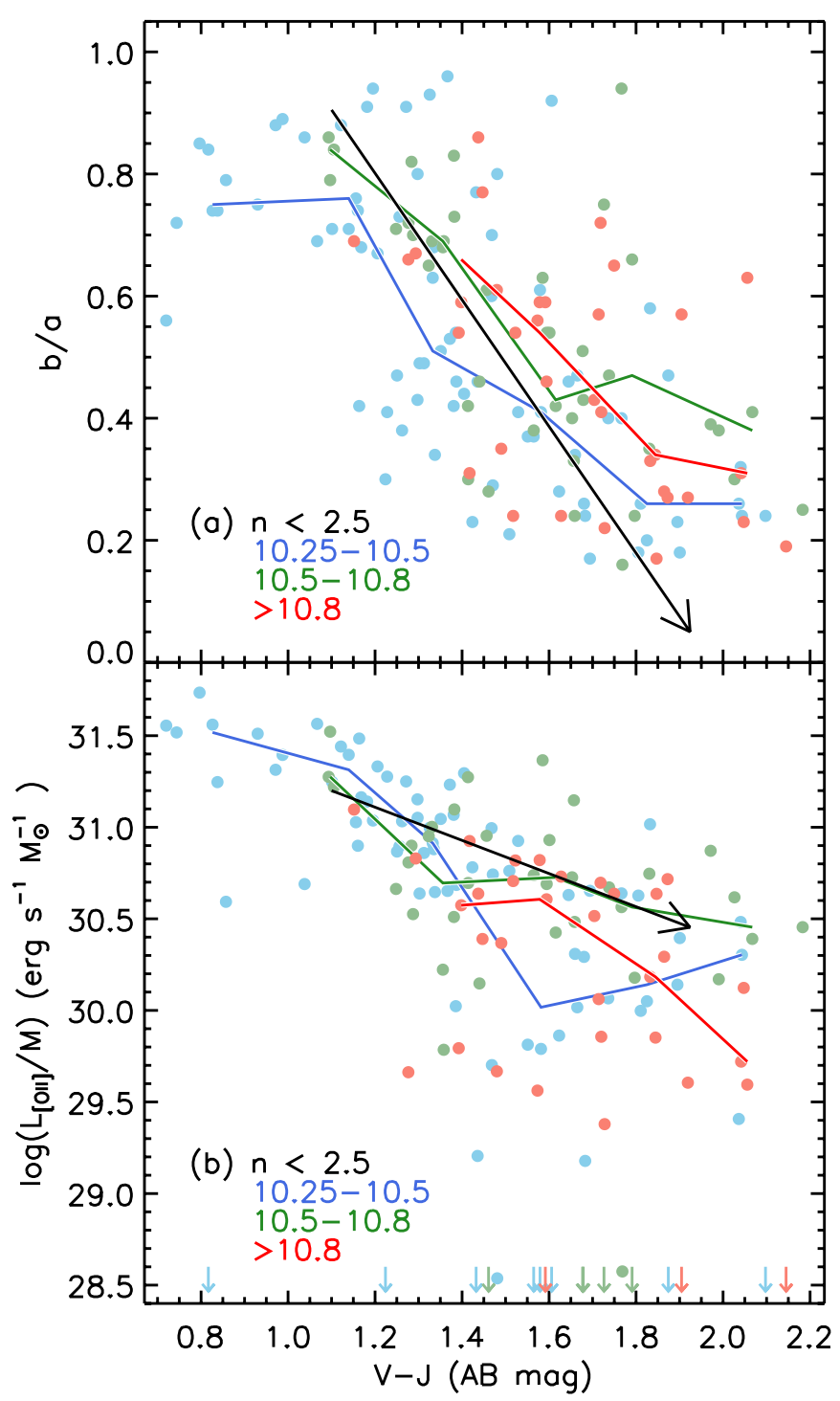

Figure 4. (a) Axis ratio, $b / a$, vs. $V-J$ for $U V J$-selected SFGs at $0.6<z<0.9$ with Sérsic index $n<2.5$. Objects are color-coded according to the same mass bins as in Figure 2. The solid colored lines indicate the median value of $b / a$ as a function of $V-J$. A clear correlation exists between $b / a$ and $V-J$. SFGs with bluer $V-J$ colors have axis ratios closer to unity, indicating that they are viewed close to face-on, while those with red colors have small axis ratios, indicating that they are viewed closer to edge-on. (b) [O II] $\lambda 3727$ luminosity, normalized by stellar mass, vs. $V-J$ for the same sample as in panel (a). Arrows on the bottom indicate objects with a lack of $[\mathrm{O}$ II $] \lambda 3727$ emission. SFGs with redder $V-J$ colors have less [O II] $] 3727$ emission, consistent with a scenario in which the light is extincted by dust in disks due to the closer to edge-on orientation. The long black arrows in panels (a) and (b) indicate the impact on $V-J$ and $L_{[\mathrm{O} \text { II }} / M$, respectively, when inclining a model Sbc galaxy from Jonsson et al. (2010) from face-on to edge-on.

(A color version of this figure is available in the online journal.)

The axis ratio serves as a proxy for the inclination. We limit our analysis to $n<2.5$ SFGs because the impact due to inclination is easier to gauge on these disk-dominated systems. Figure 4(a) shows a clear correlation between $b / a$ and $V-J$ for SFGs in all mass bins. The Pearson correlation coefficients for the low-, intermediate-, and high-mass bin are $-0.65 \pm 0.06,-0.65 \pm$ 0.10 , and $-0.53 \pm 0.13$, respectively, where the uncertainties are computed by bootstrapping. These values suggest a strong correlation between the two quantities, which provides constraints on the expected reddening in $V-J$ due to inclining a dusty disk from face-on to edge-on. Using the models of Jonsson et al.
(2010; see also Rocha et al. 2008) we find that inclining an Sbc galaxy from face-on to edge-on results in $E(V-J)=0.82 \mathrm{mag}$ (black arrows in Figure 4). Note that different dust models are likely to lead to varying degrees of reddening in $U V J$ color space. Nevertheless, this value is comparable to the observed spread in $V-J$ seen in Figure 4(a) but slightly higher than the values implied for low-redshift SFGs studied by Yip et al. (2010) and predicted by the models of Tuffs et al. (2004). The reddening due to dust could simply be lower in disks at low redshift.

Since SFGs with redder $V-J$ are more likely to be viewed closer to edge-on, one would expect light from starforming regions to be extincted. Figure 4(b) shows [O II] $\lambda 3727$ luminosities, normalized by stellar mass, versus $V-J$ for the same sample of SFGs as in Figure 4(a). The [O II] $\lambda 3727$ luminosities decline at redder $V-J$ at a fixed mass by roughly an order of magnitude. If the decline in [O II] $\lambda 3727$ luminosity was driven by a drop in the SFR, we would not expect to find many galaxies in the star-forming region of the $U V J$ diagram. For example, a factor of $\sim 6$ drop in the SFR (and in the [O II] $\lambda 3727$ luminosity) of a 5 Gyr CSF model would result in such a galaxy moving into the quiescent region after only 300 Myr. Instead, Figure 4(b) provides further support for the interpretation that the $U V J$ colors of disk-dominated SFGs are driven by an additional factor: the reddening due to the inclination, as also suggested by the model predictions of Jonsson et al. (2010, black arrow).

The correlations between $b / a$ or $\left.L_{[\mathrm{O}} \mathrm{II}\right] / M$ and $V-J$ are almost as strong at the highest masses, suggesting that inclination is an important factor in that mass regime as well. However, it is worth noting that the highest mass bin lacks objects that populate the bluer star-forming regions of the $U V J$ diagram (see Figure 3), where lower mass objects can be found. This suggests less vigorous SF in higher mass SFGs (i.e., lower specific SFR), in accord with the findings of other works (e.g., Brinchmann et al. 2004; Noeske et al. 2007), and also possibly higher levels of dust and therefore reddening in these galaxies relative to lower mass ones (see, e.g., Garn \& Best 2010) when viewed face-on.

The spread in $U V J$ colors for $n>2.5$ SFGs is also significant. However, their colors do not show a strong correlation with axis ratio. Visual inspection of these systems suggests strong dust in the redder $n>2.5$ SFGs. Thus, the dust geometry is possibly more complex or the inclination is not easily connected to the axis ratio (as can be the case for triaxial systems), leading to a poor correlation between $b / a$ and $V-J$. We note, however, that Driver et al. (2007) find strong inclinationdependent attenuation in nearby bulges.

\section{SUMMARY}

In the rest-frame $U V J$ diagram, quiescent galaxies occupy a concentrated region of color space that is distinct from the sequence of $U V J$ colors of SFGs, which extends onto the red sequence. Utilizing $H S T / \mathrm{ACS}$ imaging for the first time to study the structural parameters of galaxies in the $U V J$ diagram at $0.6<z<0.9$, we find that the commonly employed $U V J$ selection of quiescent and SFGs corresponds closely to the distinction between traditional morphological classes of early- and late-type galaxies. Meanwhile, the position of an SFG along the extended sequence of $U V J$ colors is determined to a large degree by its stellar mass and viewing inclination. Systems that are closer to edge-on generally display redder $U V J$ colors and have lower [O II] 33727 luminosity per unit mass as a consequence of the reddening from dust within the 
inclined disks. Thus, most of the $U V J$-selected SFGs on the optical red sequence are highly inclined spirals. The use of only two rest-frame colors in classifying both the recent SFHs and morphologies of galaxies will be invaluable for future studies of galaxy evolution.

We thank the referee, Richard Tuffs, for his insightful feedback. We wish to acknowledge those who have contributed to the construction and deployment of IMACS as well as Scott Burles for developing the low-dispersion prism, and the PRIMUS Collaboration for allowing us to investigate galaxies with their hardware. This research was supported by an NWO-Spinoza Grant.

\section{REFERENCES}

Baldry, I. K., Glazebrook, K., Brinkmann, J., et al. 2004, ApJ, 600, 681 Bell, E. F., Wolf, C., Meisenheimer, K., et al. 2004, ApJ, 608, 752

Blakeslee, J. P., Holden, B. P., Franx, M., et al. 2006, ApJ, 644, 30 Brammer, G. B., Whitaker, K. E., van Dokkum, P. G., et al. 2009, ApJ, 706, L173

Brammer, G. B., Whitaker, K. E., van Dokkum, P. G., et al. 2011, ApJ, 739, 24 Brinchmann, J., Charlot, S., White, S. D. M., et al. 2004, MNRAS, 351, 1151 Brown, M. J. I., Dey, A., Jannuzi, B. T., et al. 2007, ApJ, 654, 858

Bruzual, G., \& Charlot, S. 2003, MNRAS, 344, 1000

Calzetti, D., Armus, L., Bohlin, R. C., et al. 2000, ApJ, 533, 682

Chabrier, G. 2003, PASP, 115, 763

Driver, S. P., Popescu, C. C., Tuffs, R. J., et al. 2007, MNRAS, 379, 1022
Faber, S. M., Willmer, C. N. A., Wolf, C., et al. 2007, ApJ, 665, 265

Ford, H. C., Clampin, M., Hartig, G. F., et al. 2003, Proc. SPIE, 4854, 81

Garn, T., \& Best, P. N. 2010, MNRAS, 409, 421

Holden, B., van der Wel, A., Rix, H.-W., \& Franx, M. 2011, ApJ, in press (arXiv:1108.6086)

Holden, B. P., Franx, M., Illingworth, G. D., et al. 2009, ApJ, 693, 617

Jonsson, P., Groves, B. A., \& Cox, T. J. 2010, MNRAS, 403, 17

Labbé, I., Huang, J., Franx, M., et al. 2005, ApJ, 624, L81

Maller, A. H., Berlind, A. A., Blanton, M. R., \& Hogg, D. W. 2009, ApJ, 691, 394

Noeske, K. G., Faber, S. M., Weiner, B. J., et al. 2007, ApJ, 660, L47

Patel, S. G., Holden, B. P., Kelson, D. D., Illingworth, G. D., \& Franx, M. 2009a, ApJ, 705, L67

Patel, S. G., Kelson, D. D., Holden, B. P., Franx, M., \& Illingworth, G. D. 2011, ApJ, 735, 53

Patel, S. G., Kelson, D. D., Holden, B. P., et al. 2009b, ApJ, 694, 1349

Peng, C. Y., Ho, L. C., Impey, C. D., \& Rix, H.-W. 2002, AJ, 124, 266

Quadri, R. F., Williams, R. J., Franx, M., \& Hildebrandt, H. 2012, ApJ, 744, 88

Rocha, M., Jonsson, P., Primack, J. R., \& Cox, T. J. 2008, MNRAS, 383, 1281

Rudnick, G., Rix, H., Franx, M., et al. 2003, ApJ, 599, 847

Strateva, I., Ivezić, Ž., Knapp, G. R., et al. 2001, AJ, 122, 1861

Tremonti, C. A., Heckman, T. M., Kauffmann, G., et al. 2004, ApJ, 613, 898

Tuffs, R. J., Popescu, C. C., Völk, H. J., Kylafis, N. D., \& Dopita, M. A. 2004, A\&A, 419, 821

van der Wel, A. 2008, ApJ, 675, L13

Whitaker, K. E., van Dokkum, P. G., Brammer, G., et al. 2010, ApJ, 719, 1715

Williams, R. J., Quadri, R. F., Franx, M., van Dokkum, P., \& Labbé, I. 2009, ApJ, 691,1879

Williams, R. J., Quadri, R. F., Franx, M., et al. 2010, ApJ, 713, 738

Wuyts, S., Labbé, I., Franx, M., et al. 2007, ApJ, 655, 51

Yip, C., Szalay, A. S., Wyse, R. F. G., et al. 2010, ApJ, 709, 780 